\title{
Modeling, Evaluation and Control of a Road Image Processing Chain
}

\author{
Yves Lucas ${ }^{1}$, Antonio Domingues ${ }^{2}$, Driss Driouchi ${ }^{2}$, and Pierre Marché ${ }^{3}$ \\ ${ }^{1}$ Orleans University, Vision and Robotics Lab, \\ IUT Mesures Physiques 63 av. de Lattre 18020 Bourges cedex, France \\ yves.lucas@bourges.univ-orleans.fr \\ http://www. bourges. univ-orleans.fr/rech/lvr \\ ${ }^{2}$ ENSI of Bourges, Vision and Robotics Lab \\ 10 Bd. Lahitolle, 18000 Bourges, France \\ \{Antonio.Domingues, Pierre.Marche\} @ensi-bourges.fr \\ ${ }^{3}$ Pierre \& Marie Curie University, Theoretical and Applied Statistics Lab \\ 175 rue du Chevaleret 75013 Paris, France \\ driouchi@ecr.jussieu.fr \\ http://www.ccr.jussieu.fr/lsta
}

\begin{abstract}
Tuning a complete image processing chain (IPC) remains a tricky step. Until now researchers focused on the evaluation of single algorithms, based on a small number of test images and ad hoc tuning independent of input data. In this paper we explain how, by combining statistical modeling with design of experiments, numerical optimization and neural learning, it is possible to elaborate a powerful and adaptive IPC. To succeed, it is necessary to build a large image database, to describe input images and finally to evaluate the IPC output. By testing this approach on an IPC dedicated to road obstacle detection, we demonstrate that this experimental methodology and software architecture ensure a steady efficiency. The reason is simple: the IPC is globally optimized, from a large number of real images (180 out of a sequence of 30000$)$ and with adaptive processing of input data
\end{abstract}

\section{Adaptive Processing in Vision Systems}

Designing an image processing application involves a sequence of low and medium level operators (filtering, edge detection and linking, corner detection, region growing ...) in order to extract relevant data for decision purpose ( pattern recognition, classification, inspection ...). At each step of the processing, tuning parameters have a significant influence on algorithm behavior and the ultimate quality of results. As the processing power of micro computers has reached a very high level, artificial vision systems are now developed for demanding applications such as video surveillance or car driving where scene contents is uncontrolled, versatile and rapidly changing. The automatic tuning of the IPC has to be solved there, as the quality of low level vision processes should be continuously preserved to guarantee high level task robustness.

The first problem to solve in order to design adaptive vision systems is the evaluation of image processing tasks. Since a few years, researchers are interested in it and proposed rather empirical solutions $[1,2,3,4,5,6,7]$. When a ground truth is available, it is possible to compare directly this reference to the results using a specific metric. Sometimes 
no ground truth exists or remains uncertain and application experts are needed for qualitative visual assessment or empirical numerical criteria are searched for. All these methods consider only one operator at the same time $[8,9,10,11]$. However the separate tuning of each operator can not often lead to an optimal setting of the complete IPC. Moreover, image operators are generally tested on a too small number of test images, even on artificial noised images, to test algorithms efficiency. This can not replace a large real image base, for IPC testing. But how to evaluate on a great number of images a sequence of image processing operators involving numerous parameters?

A second problem remains unsolved: how to find the good tuning and so, how to adapt image processing to keep up constant quality of results ? Real time processing being executed by electronic circuits, this hardware must incorporate programmable facilities so that operator parameters can be modified at any time. Artificial retinas and also intelligent video cameras already enable the tuning of some acquisition parameters. Concerning the processing parameters, the amount of computing which is necessary to discern the effect on the results of the modification of several parameters seems at the first glance dissuasive, all the more because each separate image requires different parameters. We note that the choice of operators here still appeals to the experimenter but other works examine also the possibility of its automation $[12,13]$.

We show how to overcome these problems with an experimental approach combining statistical modeling, numerical optimization and learning. We illustrate it in the case of an IPC dedicated to line extraction for road obstacle detection.

\section{Architecture}

\subsection{General Overview}

The software is composed of six modules (Fig.1): IPC, image database, input and output evaluation, modeling and control; The IPC includes a series of low and medium level operators with configurable parameters; The database of input images is specific for an application and covering all situations; The module for IPC output evaluation measures the quality of resulting image entities. The evaluation can be supported by a ground truth or be unsupervised if empirical criteria are used instead; The module for IPC input evaluation enables the control module to tune the processing parameters for each input image. The evaluation should extract from the input images relevant

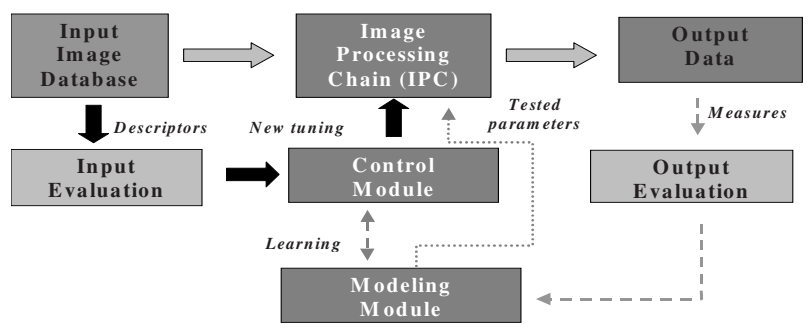

Fig. 1. Architecture of an adaptive IPC 
descriptors for IPC tuning; The modeling module; it is required firstly to model the influence of processing parameters on IPC output data and secondly to find an optimal tuning of these parameters for a given input image; Finally, a control module, based on input image description enables to provide for each input image suitable processing parameters after a learning step.

\subsection{Image Database and Evaluation}

Building a specific and exhaustive database for the target application is a crucial step to achieve good tuning of the IPC. Indeed, this database is required during modeling, optimization and control learning. From a statistical point of view, selected images should reflect the frequency of any image contents during the IPC operation and express all its versatility. Input images and output data of the IPC must be absolutely evaluated:

- Output evaluation is necessary during off-line IPC adjustment. This type of evaluation is largely discussed by researchers, even if the studies involve a single algorithm at each time. It remains a critical step, all the more because each IPC is specific and should dispose of its own evaluation criteria.

- Input evaluation is much less investigated. It is required during the IPC inline operation. Achieving adaptive and automatic IPC tuning implies to extract from input images relevant descriptors, that is to say they are closely related with IPC optimal tuning for each image. Image descriptors also enable to lower the initial dimension of the tuning problem (image size in $\mathrm{n}^{2}$ pixels), as each image pixel contributes to the tuning. Practically, a parameter vector lowers this dimension to the gray level number $(\approx \mathrm{n})$, using an histogram computed over the image.

\subsection{IPC Modeling and Optimization}

Modeling parameter influence is carried out through the design of experiments [14]. This tool is common in the industry but has been only recently introduced for machine vision applications [15]. It consists in modeling in a minimal number of trials the effects of simultaneous changes of IPC parameters.

During the experiments, the IPC is considered as a black box whose factors (tuning parameters) influence the effects (values of the criteria for output image evaluation). Tuning only one parameter at the same time can not lead to an optimal setting and testing all parameter configurations would lead to combinatorial explosion. So the goal is to identify the parameters which are really influent (high values for $\left|\mathrm{a}_{\mathrm{i}}\right|$ ) and their strong interactions (high values for $\left|\mathrm{a}_{\mathrm{ij}}\right|$ ) in relation to the effects. These notations refer to a polynomial model whose coefficients are estimated by a least square method:

$$
y=a_{0}+a_{1} x_{1}+a_{2} x_{2}+\ldots+a_{k} x_{k}+a_{12} x_{1} x_{2}+\ldots+a_{1 k} x_{1} x_{k}+\ldots+a_{1 . . k} x_{1} \ldots x_{k}
$$

A preliminary task consists in specifying for each factor an interval which bounds the experimental domain. This implies a real knowledge of how each algorithm works on image data. Practically, at each trial, every factor is set to its low or high mode, depending on -1 or +1 value in the normalized experiment matrix. So, each design of 
experiments is well defined by its experiment matrix whose line number refers to the number of trials and column number refers to the number of tested parameters. The interpretation of the experiments by variance analysis will confirm if the model obtained is really meaningful.

The amount of computing remains very high as the same trials must be repeated on a large number of test images to obtain statistical evidence. So, no optimal tuning is obtained for a given image, only an average tuning for the IPC itself. The parameter influencing significantly the quality of results are identified and the strong interactions between them are also detected, so that only the latter are considered for further IPC programming tasks.

After that, for each particular test image of the database, the optimal tuning of the IPC parameters still need to be searched. This is typically an optimization process. The average tuning obtained before provide valid initial conditions to the search process and the high and low modes of the influent parameters bound the exploration domain

To obtain the optimal parameter tuning for the IPC, we have to look for methods not based on the local gradient computing as it is not available here. The simplex method enables to explore the experimental domain and to reach maxima using a simple cost function to guide the search direction [16]. Practically, a figure of $n+1$ points of a $\mathrm{n}$-dimension space is moved and warped through geometric transformations in the parameter space, until a stop condition on the cost function is verified.

\subsection{IPC Control}

When the optimal tuning of all IPC parameters has been found for a large subset of the image database, the descriptors of these input images are computed. Together with the optimal IPC tuning parameters corresponding to the test images, it constitutes a learning base for a neural network. The other part of the database is reserved for the test of the neural network. It is a convenient tool for estimating the complex relation between the input image descriptors and the computed optimal values of the tuning parameters. At this time, if the selected descriptors are relevant for tuning purpose, the neural network should converge. Finally, after the preceding steps devoted to statistical modeling, numerical optimization and learning, the IPC is toggled into operational mode and image processing tuning parameters are continuously adapted to the characteristics of new input images.

\section{Application to a Road Image Processing Chain}

\subsection{IPC Overview}

This application is part of the French PREDIT program and has been integrated in the SPINE project (intelligent passive security ) intended to configure an intelligent airbag system in pre-crash situations. An on board multi-sensor system (EEV high speed camera + SICK scanning laser range finder) integrated in a PEUGEOT 406 experimental car classifies potential front obstacles and estimates their collision course in less than $100 \mathrm{~ms}[17,18,19]$. To respect this drastic real time constrain, low and medium image processing have been implemented in hardware with the support of MBDA 
company. It consists in two ASIC circuits [20] embedded with a DSP into an electronic board interfaced with the vehicle CAN bus. As the first tests realized by the industrial car part supplier FAURECIA demonstrated that a static tuning is ineffective against road image variability, an automatic and adaptive tuning based on the approach presented above has been successfully adopted.Eight re-configurable parameters can be modified at any time (Fig. 2).

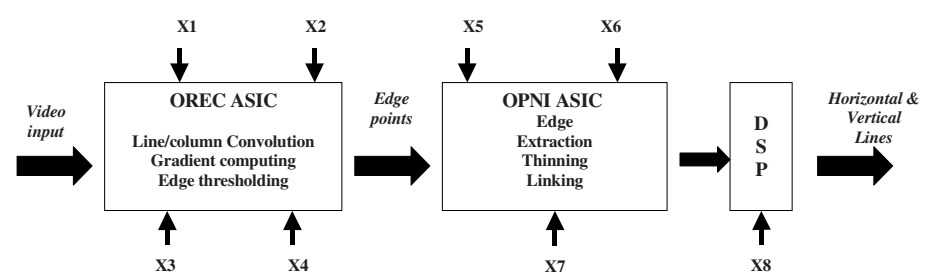

Fig. 2. Tunable parameters of the road image processing chain : Canny-Deriche filter coefficients $\left(\mathrm{X}_{1}\right)$, image amplification coefficients $\left(\mathrm{X}_{2}\right)$, edge low and high threshold values $\left(\mathrm{X}_{3}, \mathrm{X}_{4}\right)$, the number of elementary automata for contour closing $\left(\mathrm{X}_{5}\right)$, polygonal approximation threshold $\left(\mathrm{X}_{6}\right)$, small segments elimination threshold $\left(\mathrm{X}_{7}\right)$ and the approximation threshold for horizontal and vertical lines $\left(\mathrm{X}_{8}\right)$

\subsection{Output Evaluation}

The IPC should extract from the image horizontal and vertical lines (Fig.3), which, after perceptual grouping, well describe the potential obstacles in front of the experimental vehicle. Then, output evaluation is based on the number, repartition and length of these segments inside a window of interest specified by the scanning laser range finder. We have proposed a quality evaluation criteria called covering rate, which can be computed for different parameter tunings (Fig.3).

The covering rate is defined as follows: for each horizontal or vertical segment $\mathrm{S}$, we introduce a rectangular-shaped mask $\mathrm{M}_{\mathrm{S}}$, centered on this segment and whose width is

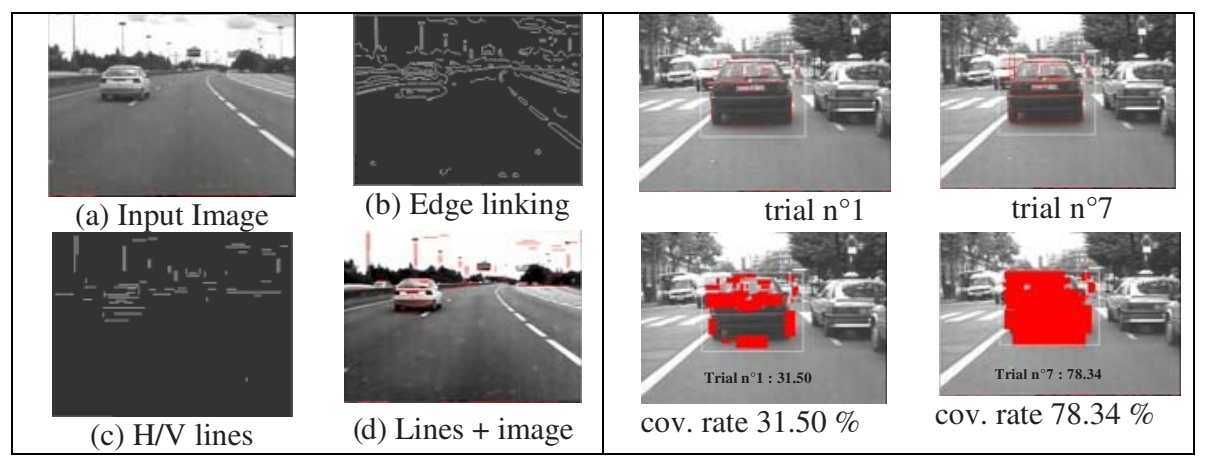

Fig. 3. H/V line Extraction (left) and IPC Output evaluation (right) 
proportional to the length of that segment. For each image pixel $(i, j)$ in the $\mathrm{W}$ window of interest ( $\mathrm{n}_{\mathrm{x}}$ and $\mathrm{n}_{\mathrm{y}}$ dimensions), we define a function $\mathrm{f}(\mathrm{i}, \mathrm{j})$ by:

$$
f(i, j)=1 \text { if } \exists S \in W \mid(i, j) \in M_{S} \text { and } f(i, j)=0 \text { otherwise }
$$

The covering rate is given by:

$$
\mathrm{r}=1 \backslash \mathrm{n}_{\mathrm{x}} \cdot \mathrm{n}_{\mathrm{y}} \cdot \Sigma_{\mathrm{i}, \mathrm{j}} \mathrm{f}(\mathrm{i}, \mathrm{j})
$$

and it is clear that $0 \leq \mathrm{r} \leq 1$. An intuitive graphical interpretation exists for the covering rate: it is simply the part of the window of interest which is covered by the superimposition of the masks associated to the set of segments detected by the IPC and so, it will be expressed in this paper as a percentage.

\subsection{Statistical Modeling}

Three design of experiments have been implemented inside the modeling module: a $2^{\mathrm{k}-\mathrm{p}}$ factorial fractional design with 16 trials [21] to select the really influent parameters $\left(\mathrm{X}_{1}\right.$, $\mathrm{X}_{6}, \mathrm{X}_{8}$ ), a Rechschaffner design [22] with 37 trials and finally a quadratic design with 27 trials, by adding an intermediate zero mode to detect non linearity. By using two modes for the tuning of each parameter (Tab.2), 256 different IPC output can be compared from any given input image.

To give an example, we present just below the experiment matrix and the covering rate for the set of trials of the first design of experiments (Tab.1). These designs have been tested on 180 input images selected from a video sequence of over 30000 city and motorway frames. Selected images should correspond to representative road scenarios

Table 1. Experiment matrix (fact. $2^{8-3}$ design ) and averaged outputs. It can be observed that the so called generators are $\mathrm{X}_{5}=2.3 .4, \mathrm{X}_{6}=1.3 .4, \mathrm{X}_{7}=1.2 .3, \mathrm{X}_{8}=1.2 .4$, where the numbers refer to the columns 1 to 4

\begin{tabular}{cccccccccc}
\hline & $\mathbf{X}_{\mathbf{1}}$ & $\mathbf{X}_{\mathbf{2}}$ & $\mathbf{X}_{\mathbf{3}}$ & $\mathbf{X}_{\mathbf{4}}$ & $\mathbf{X}_{\mathbf{5}}$ & $\mathbf{X}_{\mathbf{6}}$ & $\mathbf{X}_{\mathbf{7}}$ & $\mathbf{X}_{\mathbf{8}}$ & $\mathbf{r}(\mathbf{\%})$ \\
\hline $\mathbf{1}$ & -1 & -1 & -1 & -1 & -1 & -1 & -1 & -1 & 35.535 \\
$\mathbf{2}$ & -1 & -1 & -1 & 1 & 1 & 1 & -1 & 1 & 40.310 \\
$\mathbf{3}$ & -1 & -1 & 1 & -1 & 1 & 1 & 1 & -1 & 27.859 \\
$\mathbf{4}$ & -1 & -1 & 1 & 1 & -1 & -1 & 1 & 1 & 42.436 \\
$\mathbf{5}$ & -1 & 1 & -1 & -1 & 1 & -1 & 1 & 1 & 47.328 \\
$\mathbf{6}$ & -1 & 1 & -1 & 1 & -1 & 1 & 1 & -1 & 30.284 \\
$\mathbf{7}$ & -1 & 1 & 1 & -1 & -1 & 1 & -1 & 1 & 44.034 \\
$\mathbf{8}$ & -1 & 1 & 1 & 1 & 1 & -1 & -1 & -1 & 37.743 \\
$\mathbf{9}$ & 1 & -1 & -1 & -1 & -1 & 1 & 1 & 1 & 46.517 \\
$\mathbf{1 0}$ & 1 & -1 & -1 & 1 & 1 & -1 & 1 & -1 & 40.469 \\
$\mathbf{1 1}$ & 1 & -1 & 1 & -1 & 1 & -1 & -1 & 1 & $\mathbf{5 0 . 6 8 0}$ \\
$\mathbf{1 2}$ & 1 & -1 & 1 & 1 & -1 & 1 & -1 & -1 & 33.464 \\
$\mathbf{1 3}$ & 1 & 1 & -1 & -1 & 1 & 1 & -1 & -1 & 35.169 \\
$\mathbf{1 4}$ & 1 & 1 & -1 & 1 & -1 & -1 & -1 & 1 & 49.255 \\
$\mathbf{1 5}$ & 1 & 1 & 1 & -1 & -1 & -1 & 1 & -1 & 39.715 \\
$\mathbf{1 6}$ & 1 & 1 & 1 & 1 & 1 & 1 & 1 & 1 & 44.842 \\
\hline
\end{tabular}


and reveal enough different input descriptors. A statistical model has been deduced and validated by measuring R-Square (0.897) and Mallow C(p) indicator . It is given by :

$$
Y=40.2+2.06 X_{1}+0.74 X_{2}-2.47 X_{6}+5.30 X_{8}-0.92 X_{1} X_{2}+0.95 X_{6} X_{8}
$$

Higher order interactions between the parameters are practically neglected and non significant parameters should not generate significant interactions. The covering rates obtained for the different trials provides an average tuning for the IPC parameters. This static tuning can not be optimal for each given input image but it allows to initialize the optimization of the Nelder \& Mead algorithm based on the simplex method. This algorithm then computes all the parameter optimal values corresponding to each tested input image.

Table 2. Modes for all the design of experiments

\begin{tabular}{cclc}
\hline Factor & Parameter & \multicolumn{2}{c}{ Mode } \\
\hline $\mathrm{X}_{1}$ & Canny-Deriche $(\alpha)$ & 0.5 & 1 \\
$\mathrm{X}_{2}$ & Image amplification & 33 & 63 \\
$\mathrm{X}_{3}$ & Edge low threshold & 5 & 15 \\
$\mathrm{X}_{4}$ & Edge high threshold & 15 & 30 \\
$\mathrm{X}_{5}$ & Elementary automata (edge closing) & 26 & 30 \\
$\mathrm{X}_{6}$ & Polygonal approx. threshold & 5 & 6 \\
$\mathrm{X}_{7}$ & Small segments suppression threshold & 5 & 10 \\
$\mathrm{X}_{8}$ & Horizontal/ Vertical approx. threshold & 1 & 3 \\
\hline
\end{tabular}

\subsection{Input Evaluation}

Before to start the learning of the control module, input descriptors should be found to characterize input images. The homogeneity histogram [23] of the input image has been selected to take in account regions with uniform shade (vehicle paintings) as well as homogeneous texture (road surface) (Fig.4). The homogeneity measure combines two

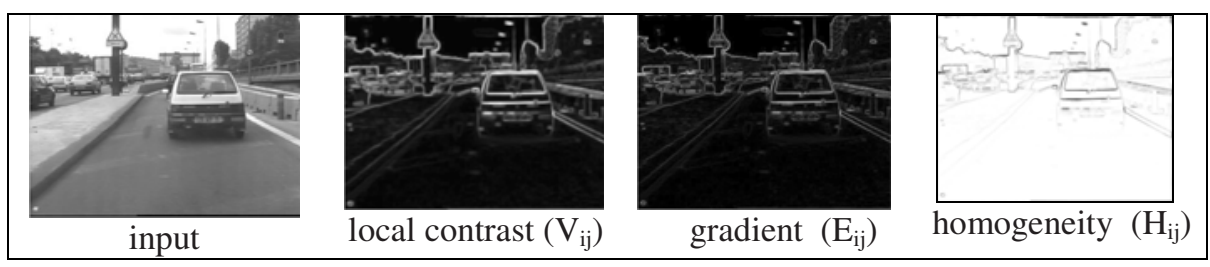

Fig. 5. Homogeneity measure. Each pixel (i,j) with a $H_{i j}$ measure verifying $H_{i j}>0.95$ is taken in account in the histogram computed on the 256 gray levels of the input image

local criteria: the local contrast $\sigma_{\mathrm{ij}}$ in a $\mathrm{d} \mathrm{x} \mathrm{d}(\mathrm{d}=5)$ window centered on the current pixel (i,j) using $\mu_{i j}$, the average of the gray levels computed inside the same window and a gradient measure $e_{i j}$ in another $\mathrm{x} t(\mathrm{t}=3)$ window. The measure of intensity variations 
$e_{i j}$ around a pixel $(\mathrm{i}, \mathrm{j})$ is computed by Sobel operator based on the components of the gradient at pixel $(\mathrm{i}, \mathrm{j})$ in $\mathrm{x}$ and $\mathrm{y}$ directions. These measures are normalized using $\mathrm{V}_{\mathrm{ij}}=$ $\sigma_{\mathrm{ij}} / \max \sigma_{\mathrm{ij}}$ and $\mathrm{E}_{\mathrm{ij}}=\mathrm{e}_{\mathrm{ij}} / \max \mathrm{e}_{\mathrm{ij}}$. The homogeneity measure is finally expressed by:

$$
H_{i j}=1-E_{i j} \cdot V_{i j}
$$

\subsection{IPC Control}

We have used a simple multi layer perceptron as a control module. It is composed of 256 input neurons (homogeneity histogram levels over the 256 gray levels ), 48 hidden neurons (maximum speed convergence during the learning) and 3 output neurons corresponding to the 3 influent tuning parameters of the IPC. During the learning step carried out on $75 \%$ of the 180 input images, we can observe the decrease of the mean absolute error (MAE) between optimal parameters and that computed by the network. (convergence over 400 iterations)

\section{Results}

The separate validation set is constituted by the $25 \%$ remaining test images. An essential task is to control on these images that the tuning parameter computed by the network, not only are close enough to the optimal values, but also enable to obtain really good results at the IPC output, that is to say line groups are well detected (covering rate is close to that derived from simplex algorithm) (Tab. 3).

Table 3. Neural network performance

\begin{tabular}{cccccc}
\hline & \multicolumn{3}{c}{ Learning } & \multicolumn{2}{c}{ Test } \\
\hline Neural & Hidden & Parameter & Covering rate & Parameter & Covering rate \\
network & neurons & MAE $(\%)$ & Abs. error $(\%)$ & MAE $(\%)$ & Abs. error $(\%)$ \\
RN3 & $\mathbf{4 8}$ & 1.4 & 8.06 & 23.7 & 9.53 \\
RN8 & $\mathbf{8 0}$ & 0.8 & 3.55 & 28.6 & 13.17 \\
\hline
\end{tabular}

We can notice that the neural network only based on influent tuning parameters (RN3) is the most robust during the test step although errors are larger during the learning step. We compare in (Tab.4) the covering rates (output quality evaluation) averaged on the set of 180 test images extracted from the 30000 image sequence, depending on the tuning process adopted. Without any adaptive tuning facility (static averaged tuning issued from the design of experiments), the results are low; when the best trial obtained from a design of experiments is used for the tuning, the results are high enough but this method can not be applied in real time situations; the results obtained with the simplex (SPL) method are naturally optimal but the price for that is the prohibitive time for parameter space exploration; finally, the neural networks (RN) obtain high results, especially the 3 output network, with a negligible computing cost $(\approx$ computation of the input image descriptors). 
Table 4. Comparison of several tuning methods

\begin{tabular}{lclllllll}
\hline & Static & RN8 & RN3 & $\begin{array}{l}\text { Fact. } \\
\text { design }\end{array}$ & $\begin{array}{l}\text { Rech. } \\
\text { design }\end{array}$ & $\begin{array}{l}\text { Quad. } \\
\text { design }\end{array}$ & SPL8 & SPL3 \\
\hline Mean Cov. Rate(\%) & 34.84 & 45.17 & $\mathbf{4 9 . 6 4}$ & 50.68 & 51.06 & 55.02 & 58.34 & 59.17 \\
Cost by Image & 0 & $\begin{array}{l}\text { Hist. } \\
\text { Comp. }\end{array}$ & $\begin{array}{llll}\text { Hist. } \\
\text { Comp. }\end{array}$ & $\begin{array}{l}16 \\
\text { trials }\end{array}$ & $\begin{array}{l}37 \\
\text { trials }\end{array}$ & $\begin{array}{l}27 \\
\text { trials }\end{array}$ & $\begin{array}{l}100 \\
\text { trials }\end{array}$ & $\begin{array}{l}60 \\
\text { trials }\end{array}$ \\
\hline
\end{tabular}

We have intentionally mentioned in this table the results obtained for a 8 parameter tuning: we can easily verify that the tuning of the 5 parameters considered little influent by the design of experiments, is useless.

\section{Conclusion}

These interesting results obtained in the context of an image processing chain (IPC) dedicated to road obstacle detection highlight the interest of the experimental approach for the adaptive tuning of an IPC. We demonstrated that only three parameters have to be precisely tuned and that with IPC knowledge combined with input image description an automatic tuning can be obtained in real time for each sequence image. The main reasons for this efficiency are simple: contrary to previous works, the IPC is globally optimized, from a great number of real test images, and by adapting image processing to each input image. We are currently testing this approach on other applications in which the image typology, image processing operators and data evaluation criteria for inputs as well as outputs are still specific. This should enable us to unify and generalize this methodology for better IPC performance.

\section{Acknowledgments}

This research program is supported by the French PREDIT Program and by Europe FSE grant.

\section{References}

1. R.M. Haralick, "Performance characterization protocol in computer vision ", ARPA Image Understanding Workshop, Monterey, CA, 667-673, 1994.

2. P.Courtney, N.Thacker, A. Clark "Algorithmic modeling for performance evaluation" Workshop on perf. characteristics of vision algorithms Cambridge, April 19 1996- 13p.

3. W. Forstner, "10 Pros and cons against performance characterization of vision algorithms", in Workshop on Performance Characteristics of Vision Algorithms, April 1996.

4. Kevin W. Bowyer, P. Jonathon Phillips "Empirical Evaluation Techniques in Computer Vision” June 1998, Wiley-IEEE Computer Society Press ISBN: 0-8186-8401-1 262 pages

5. P. Meer, B. Matei, K. Cho, “Input Guided Performance Evaluation “, Theoretical Foundations of Computer Vision, pp. 115-124, 1998.

6. I.T. Phillips and A.K. Chhabra, "Empirical Performance Evaluation of Graphics Recognition Systems", IEEE PAMI, vol. 21, pp. 849-870, 1999. 
7. J. Blanc-Talon, V. Ropert "Evaluation des chaînes de traitement d'images" Revue Scientifique et Technique de la Défense ${ }^{\circ} 462000$ p.29-38

8. S. Philipp-Foliguet, “Evaluation de la segmentation “, ETIS, Cergy-Pontoise, Mars 2001.

9. N.Sebe, Q Tian, E. Loupias, M. Lew, T. Huang "Evaluation of salient point techniques" CIVR 02 July 10-15 2002 London

10. P.Rosin, E. Ioannidis "Evaluation of global image thresholding for change detection " Pattern Recognition Letters 24 (2003) 2345-2356

11. Y. Yitzhaky, E. Peli "A method for objective edge detection evaluation and detection parameter selection" IEEE PAMI vol 25 nº Aug. 2003 p.1027-1033.

12. V. Ropert, "Proposition d'une architecture de contrôle pour un système de vision ", Thèse de l'Université René Descartes (Paris 6), France, Décembre 2001.

13. I. Levner, V. Bulitko "Machine learning for adaptive image Interpretation" The Sixteenth Innovative Appl. of Art. Intell. Conf. (IAAI-04) July 27-29, 2004 San Jose, (CA) USA- 7p.

14. P. Schimmerling, J-C. Sisson, A. Zaïdi, "Pratique des Plans d'Expériences", Lavoisier Tec \& Doc, ISBN 2-743-00239-5, 1998.

15. S. Treuillet, “Analyse de l'influence des paramètres d'une chaîne de traitements d'images par un plan d'expériences “, $19^{e}$ colloque GRETSI'03, 8-11 sept. 2003.

16. Margaret H. Wright The Nelder-Mead Simplex Method: Recent Theory and Practice Int. Symposium on Mathematical Programming Lausanne, EPFL, August 24-29, 1997

17. A. Domingues, Y. Lucas, D. Baudrier, P. Marché, "Détection et suivi d'objets en temps Détection et suivi d'objets en temps réel par un système embarqué multi capteurs “, GRETSI'01, Toulouse, Septembre 2001

18. A. Domingues, "Système embarqué multicapteurs pour la détection d'obstacles routiers Développement du prototype et réglage automatique de la chaîne de traitement d'images ", Thèse de l'Université d'Orléans, France 15 Juillet 2004.

19. Y. Lucas, A. Domingues, M. Boubal, P. Marché, "Système de vision embarqué pour la détection d'obstacles routiers " Techniques de l'Ingénieur - Recherche Innovation. 2005

20. P. Lamaty, “ Opérateurs de niveau intermédiaire pour le traitement temps réel des images “, Thèse de Doctorat, France 2000.

21. A. Fries, J. Hunter, "Minimum aberration $2^{\mathrm{k}-\mathrm{p}}$ designs", Technometrics, vol. 22, pp. 601-608, 1980.

22. R. L. Rechtschaffner, "Saturated fractions of 2 n and 3 n factorial designs", Technometrics, 9 , pp. 569-575, 1967.

23. H. Cheng, Y. Sun, "A hierarchical approach to color image segmentation using homogeneity”, in IEEE transactions on image processing 9(12): 2071-2082, 2000. 